\title{
NARRATIVAS ÉTICAS, SIMBÓLICAS Y POLÍTICAS \\ ASOCIADAS AL CONSUMO DE ALIMENTOS AGROECOLÓGICOS. UN ESTUDIO DE CASO
}

\author{
MARLON JAVIER MÉNDEZ SASTOQUE ${ }^{1}$ \\ FAUSTO MANUEL BONILLA ORREGO ${ }^{2}$
}

\begin{abstract}
Recibido el 18 de mayo de 2012 y aprobado el 30 de noviembre de 2012
\end{abstract}

\section{RESUMEN}

Este artículo tiene como fin precisar y analizar algunas de las cuestiones éticas, simbólicas y políticas asociadas al consumo de alimentos agroecológicos, implícita y explícitamente manifiestas por los consumidores al optar por este tipo de alimentos. Los hallazgos dan cuenta de las narrativas mediante las cuales los consumidores justifican para sí y para los demás dicha elección preferencial. Empíricamente, lo expuesto se sustenta en el análisis de los razonamientos y prácticas de algunos frecuentadores permanentes y ocasionales del Mercado Agroecológico de Manizales, espacio de encuentro e interacción auspiciado por el Jardín Botánico de la Universidad de Caldas. Los resultados se organizan en torno a cinco ejes relacionales: a) consumo agroecológico y responsabilidad socioambiental, b) consumo agroecológico y práctica ciudadana, c) consumo agroecológico y diferenciación identitaria, d) consumo agroecológico, afectividad y cuidado, e) consumo agroecológico y expectativas alimentarias, y f) consumo agroecológico y evocación sensorial. El artículo concluye reforzando el acto de preferir alimentos agroecológicos como un proceso de apropiación de los significados y valores contenidos en cada producto, a la luz de las representaciones construidas por los consumidores.

\section{PALABRAS CLAVE:}

Consumo agroecológico, producción agroecológica, responsabilidad socioambiental, ética del consumo, sociología del consumo. 


\title{
ETHICAL, SYMBOLIC, AND POLITICAL NARRATIVE ASSOCIATED TO THE AGROEACOLOGICAL FOOD CONSUMPTION. A CASE STUDY.
}

\begin{abstract}
This paper aims to clarify and discuss some of the ethical, symbolic, and political questions associated to the concumption of agroecological food products implicitly and explicitly reported by consumers through their option for.this type of food. The findings account for narratives through which consumers justify for them and for others such preferential election. Empirically, what is presented above is based on the analysis of rationale and practices of some permanent and occasional frequenters of the Manizales Agroecology Market which is an, interaction and meeting space sponsored by the Botanical Garden of Universidad de Caldas. The results are organized around five relational axes: a) agroecological consumption and environmental responsibility, b) agroecological consumption and citizenship practice, c) agroecological consumption and social differentiation, d) agroecological consumption, affectivity and care, e) agroecological consumption and food expectations, and f) agroecological consumption and sensory evoking. The article concludes emphasizing the act of prefering agroecological food as an appropriation process of meanings and values contained in each product, in the light of representations constructed by the consumers.
\end{abstract}

\section{KEY WORDS:}

Agroecological consumption, agroecological production, environmental responsibility, ethics of consumption, consumption sociology

\section{INTRODUCCIÓN}

Quienes tenemos interés en las relaciones de mercado como referente contemporáneo para la comprensión de las actuales dinámicas sociales, reconocemos la existencia de un vacío en el conocimiento en referencia a la siguiente temática: la producción y el consumo de alimentos agroecológicos como referente de nuevas sociabilidades, más allá de las implicaciones técnicas o de injerencia comercial asociadas a este tipo de bienes. En contextos en los que prima la producción sobre el consumo, visión que usualmente prevalece en escenarios formativos como las facultades de ciencias agropecuarias, el conocimiento 
generado sobre ello se centra en aspectos que privilegian las cuestiones productivas y mercadotécnicas, desconociendo, en la mayoría de los casos, lo asociado a las prácticas de consumo y su significación simbólica, ética y política.

Investigaciones recientes como la de Gulh (2009), Parrado y Montoya (2007), entre otras, son una muestra de ello. Gulh (2009), autor que centra la mirada en la producción certificada de café en Colombia, concluye que adoptar tecnologías y sistemas productivos de reducido impacto ambiental constituye una apreciable estrategia de reactivación y diversificación productiva para los pequeños productores, omitiendo en su análisis el papel de los consumidores como parte integral de esa estrategia, al ser éstos los demandantes de tal tipo de productos. Por su parte, centrados en la cuestión mercadotécnica, Parrado y Montoya (2007) dan cuenta de las características de consumo de vegetales orgánicos (centrando el estudio en la determinación de preferencias, frecuencias y lugares de compra), señalando algunas tendencias útiles para la definición de estrategias de mercadeo agropecuario. En este caso, los consumidores son básicamente reducidos a su papel de compradores en sentido estricto, desconociendo o prestando mínima atención a las ideas y valores que sustentan el acto de preferir y adquirir esos bienes sobre otros.

En síntesis, se trata de estudios que, centrados en la producción y el mercadeo agropecuario, dejan al margen una arista importante del encadenamiento agroalimentario: las ideas y representaciones que soportan las elecciones realizadas por sujetos activos y pensantes, quienes, a partir de sus decisiones conscientes y reflexivas de consumo, dinamizan y sostienen dicho nicho específico de mercado.

En este contexto, buscando contribuir al conocimiento disponible acerca del consumo agroecológico y pretendiendo ir más allá de los análisis del perfil de los consumidores en su calidad de simples compradores o demandantes de alimentos, la investigación que dio origen a este artículo enfatiza un asunto particular, a saber, las funciones simbólicas y sociales atribuibles al consumo de alimentos agroecológicos, a la luz de lo expuesto por los propios consumidores. Específicamente, el objetivo del estudio fue reconocer, analítica e interpretativamente, la manera en que los consumidores de alimentos agroecológicos incorporan cuestiones éticas, simbólicas y políticas en sus elecciones de consumo, a partir de la respuesta a la siguiente pregunta ¿Qué narrativas son construidas por ellos para justificar para sí mismos y los otros la opción por este tipo de productos? El escenario 
empírico de indagación corresponde al Mercado Agroecológico mensualmente organizado por el Jardín Botánico de la Universidad de Caldas en el municipio de Manizales, Colombia (Figura 1).

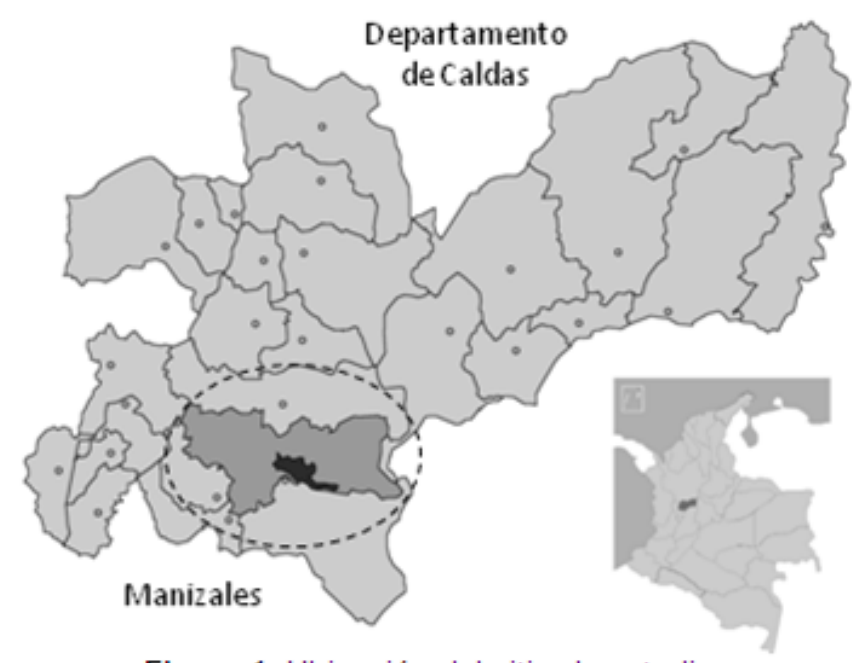

Figura 1. Ubicación del sitio de estudio.

Fuente: http://fr.wikipedia.org/wiki/Fichier:MunsCaldas_Manizales.png\#filelinks

Los postulados teórico-conceptuales que orientaron tanto el planteamiento del problema como el levantamiento y el análisis de resultados, todos amparados en la sociología del consumo, se sintetizan a continuación.

En términos generales, el acto de comprar, de elegir qué comprar, de decidir qué consumir y qué no adquirir o rechazar, es un acto cotidiano de auto-expresión que comunica acerca de lo que los consumidores, en su papel de actores sociales, son o desearían ser (Campbell, 2006). En este sentido, podría plantearse que los sujetos consumen con una intención comunicativa. Los bienes adquiridos representarían una forma de visibilizar lo que se es con relación a lo que no se es, creando una definición propia basada en la diferencia.

Sobre este supuesto, cuando los actores sociales optan por comprar y consumir productos ecológicos, dándoles prelación sobre otros de distinta índole (alimentos producidos convencionalmente, recurriendo al uso excesivo de insumos químicos, por ejemplo), estarían, hasta cierto punto, exteriorizando o poniendo en manifiesto parte de aquello que ellos mismos son o intentan ser. "Personas comprometidas con la cuestión ambiental", "sujetos practicantes de la justicia social", "individuos adeptos a la alimentación sana", "gente en contra de un modelo productivo basado en el uso desmedido de agroquímicos", entre otras posibles afirmaciones, constituyen ejemplos de 
autodefiniciones que informarían sobre aquello que los distingue y califica.

En esta vía, a autoafirmaciones identitarias comunes, como las asociadas a género, lugar de pertenencia y profesión (V.gr. "soy hombre", "soy mujer", "soy joven", "soy colombiano", "soy ama de casa"), se sumarían otras definidas a partir de los gustos y elecciones particulares de consumo, e.g. "soy un consumidor de productos ecológicos", "soy un consumidor responsable", referentes que a su vez podría calificar al individuo como "un aliado del comercio justo", "un defensor de la agricultura familiar", "un detractor del trabajo esclavo e infantil", un estudioso amateur de los sistemas de producción agropecuaria, "un individuo respetuoso de los saberes ancestrales".

Sobre esta lectura, aquellos productos consumidos, además de resultar útiles en los procesos de reproducción física o social (como podría ser el caso de los alimentos ecológicos consumidos por sus características saludables y nutricionales), también auxiliarían a los consumidores en la construcción de sus propias identidades (Barbosa \& Campbell, 2006). Es decir, además de apropiarse de su valor de uso (de sus propiedades como alimentos en sí), al consumirlos ellos también conseguirían apropiarse de los significados que crea su consumo, marcaciones de prestigio, pertenencia, adecuación o no a las reglas socialmente aceptadas, reconocimiento y diferenciación social, entre otros (Warde, 2005; Douglas \& Isherwood, 2006; Méndez, 2007).

Ilustrando lo anterior, al consumir alimentos ecológicos (hortalizas producidas por agricultores de economía campesina, bajo un esquema de producción solidaria y con tecnologías limpias, por ejemplo), además de satisfacer sus necesidades alimentarias, esos mismos consumidores podrían estar su vez "ganando" un estatus social específico. En este caso sería el estatus de "consumidores ecológicos", distinción socialmente conferida a aquellos grupos e individuos caracterizados por incorporar la preocupación socioambiental a la mayoría de sus actos de consumo, lo que les permitiría distinguirse de otros individuos orientados por otras lógicas de consumo.

En dichas circunstancias, consumir productos ecológicos sería algo así como un medio no verbal para expresar públicamente aptitudes y valores de consumo incorporados a la práctica cotidiana de vida en sociedad. "Responsabilidad social y ambiental", "compromiso con la justicia social" y "respeto a la naturaleza", entre otros, harían parte de esa lista de comportamientos distintivos, usados no sólo para autoafirmarse diferentes, sino también 
para hacer notar a otros la existencia de formas alternativas de consumo y posicionamiento ante el mundo.

Se trataría de una concesión socialmente mediada, útil para establecer fronteras entre "aquellos comprometidos con el consumo ecológico", "los que delegan la responsabilidad socioambiental en otros" y "aquellos indiferentes al consumo consciente", P. ej. , donde, siguiendo a García Canclini (1999), cada una de las facciones formadas a partir de las variadas elecciones de consumo definiría lo que es públicamente valioso para sí, así como las formas de integrarse y distinguirse dentro de la sociedad en que se vive. En estos términos, el mismo autor plantea que al consumir también se piensa, es decir, se elige y se otorga sentido social a dicha acción, constituyendo el acto de consumir una forma de ejercicio ciudadano. En este sentido, como argumenta Portilho (2005), el consumo podría ser asumido como una vía de participación política, en otras palabras, como una manera de materializar tornando públicos valores y preocupaciones ambientales como sociales, en este caso usando como forma de expresión y acción política el consumo de alimentos agroecológicos.

Partiendo de lo anterior, resultaría coherente plantear que, como mecanismo de expresión social, el consumo ecológico podría surgir como una forma de activismo. De acuerdo con Stolle, et al. (2005), en un entorno social diverso y disonante, donde hay cabida para las más distintas racionalidades, el consumo de productos basado en criterios éticos y políticos constituiría una posibilidad de acción y toma de postura frente a ciertas prácticas de producción y consumo dominantes. Como muestran distintos autores, históricamente, convocar a los consumidores a no comprar determinados productos, a abstenerse de adquirir bienes producidos por una u otra empresa o producidos siguiendo prácticas con las que no están de acuerdo, por ejemplo, ha constituido, durante largo tiempo, un instrumento de ejercicio ciudadano (ver Blee, 1985; Liden, 1994; Cohen, 2001, entre otros). En primera instancia, se trata acciones conducentes a ejercer presión económica por cambios de índole socio y técnico ambiental, forzando a adoptar nuevas prácticas productivas.

Por otro lado, pasando a un plano enteramente cotidiano, como complemento a la acción política del consumo, vale la pena considerar la lógica afectiva implícita en la acción de consumir. Para autores como Miller (2002), comprar puede ser visto un acto de amor o cuidado hacia los otros. En la vida diaria, las compras (aquello que se adquiere para brindar a otros, a los miembros de la propia familia o 
para el consumo personal) representan un medio básico para la construcción de relacionamientos afectivos y para demostrar compromiso por el bienestar propio y ajeno. Bajo tal óptica, seleccionar y comprar el mejor producto pensando en el bienestar y el gusto de esas personas que se quieren, así como comprar y consumir aquello producido por quienes, por distintas circunstancias, estimamos y valoramos, constituyen actos de afecto. En el caso del consumo ecológico, adquirir alimentos orgánicos pensando en la salud de los hijos; ir de compras al mercado ecológico en reconocimiento a la labor de aquellos que se esfuerzan por producir sanamente; seleccionar productos con atributos éticos entre toda la gama de similares disponibles en el estante del supermercado, entre muchos otros actos cotidianos, constituyen ejemplos de lo expuesto.

En síntesis, se trata de acciones no siempre percibidas, usualmente presentes en las prácticas cotidianas de consumo, que contribuyen a consolidar el sentido de lo que, a manera de hipótesis, significa consumir productos agroecológicos.

\section{METODOLOGÍA}

Se desarrolló un trabajo etnográfico que involucró observación participante en combinación con conversaciones informales y entrevistas semi-estructuradas sostenidas con los consumidores concurrentes al Mercado Agroecológico de Manizales, evento organizado el tercer sábado de cada mes por el Jardín Botánico de la Universidad de Caldas, en el parqueadero de la Facultad de Ciencias Jurídicas y Sociales. Esta levantamiento aconteció entre febrero y marzo del 2010 , período en que se interactuó con 43 consumidores. En dicho espacio fueron realizadas 19 entrevistas a profundidad.

Con la observación participante se valoró la forma en que los distintos actores (consumidores y productores) interactúan entre sí en el mercado agroecológico (en este caso asumido como espacio de sociabilidad y no únicamente de intercambio comercial), identificando justificaciones y motivaciones declaradas en referencia a la opción por alimentos ecológicamente producidos, así como rasgos distintivos de la interacción establecida entre las partes (interés en el otro, su quehacer y sus motivaciones, respeto por su labor productiva, confianza en el producto ofrecido, proximidad e intimidad en el trato, entre otros ítems). Considerando la riqueza de los detalles, se optó por elaborar y mantener un minucioso diario de campo. 
Las entrevistas y conversaciones informales versaron sobre los siguientes asuntos: el inicio en el consumo de este tipo de alimentos, incluyendo las razones que condujeron a ello; los valores atribuidos a los alimentos agroecológicos (prestando atención a las diversas perspectivas desde las cuales los interlocutores hacían sus planteamientos); las visiones acerca de lo que significa y provoca (individual y colectivamente) optar por éstos; la importancia dada a contar en el ámbito local con un espacio como el mercado agroecológico, entre los principales. Acogiendo las recomendaciones expuestas por Jodelet (2006), durante las interlocuciones se procuró comenzar con preguntas más concretas, factuales y relacionadas con la práctica específica de consumo, para posteriormente pasar a preguntas que implicaron reflexiones más abstractas, emociones y juicios de valor sobre esta misma experiencia.

El perfil de los entrevistados, en términos generales, es el siguiente: hombres y mujeres entre los 22 y 63 años de edad, con diferentes ocupaciones: amas de casa (en su mayoría casadas y con hijos), estudiantes de pregrado y postgrado, profesores (universitarios, de enseñanza media y básica primaria), agrónomos, médicos, abogados, sociólogos, médicos veterinarios, administradores del medio ambiente, antropólogos. Se trata de consumidores de buen nivel educacional, clasificables como de clase media, habitantes de barrios considerados por ellos como cercanos al Mercado Agroecológico. Estos consumidores pueden diferenciarse entre permanentes (frecuentadores constantes del mercado desde hace por lo menos dos años) y consumidores ocasionales (quienes tienen conocimiento del mercado y lo visitan de manera esporádica). Con relación al factor tiempo, todos expresaron preferencia por los alimentos agroecológicos desde hace por lo menos dos años.

Como factor congregante, hecho corroborado desde las indagaciones iniciales, la mayoría de entrevistados comparten algunos valores éticos, políticos, ideológicos y ecológicos con relación a la responsabilidad socioambiental y el significado de sus elecciones justificadas de consumo, circunstancia que permite observarlos como grupo. Sin embargo, resultaría vago decir que los frecuentadores del Mercado Agroecológico conforman una comunidad, en el sentido convencional, sin que esto quiera decir que se trata apenas de una serie de individuos cuya acción es individualizada y segmentada. Acogiendo lo expuesto por Miller (2002), apreciación también realizada por Portilho (2009) y Castañeda (2010) para casos brasileños, se trataría de una "comunidad imaginaria", donde sus integrantes refuerzan mutuamente sus estilos de vida, ideas y valores, compartiendo una esfera pública común. 
Para el caso específico de estudio, es necesario considerar que, dada la frecuencia mensual del mercado, la disponibilidad constante de alimentos agroecológicos dificulta el acceso permanente a ellos por parte de los consumidores. No obstante, para efectos de la investigación, es la convicción de adquirirlos cada vez que sea posible lo que marca la pauta en términos de consumo, es decir, en la construcción del referente "consumidores (agro)ecológicos".

Volviendo a las labores de campo, como complemento de las entrevistas y conversaciones informales, también se recurrió al levantamiento de información contenida en documentos escritos producidos por los organizadores del mercado (plegables, invitaciones a participar del mercado y a otras actividades asociadas a la visión agroecológica), incluyendo los pendones publicitarios allí expuestos (ver Figura 2).

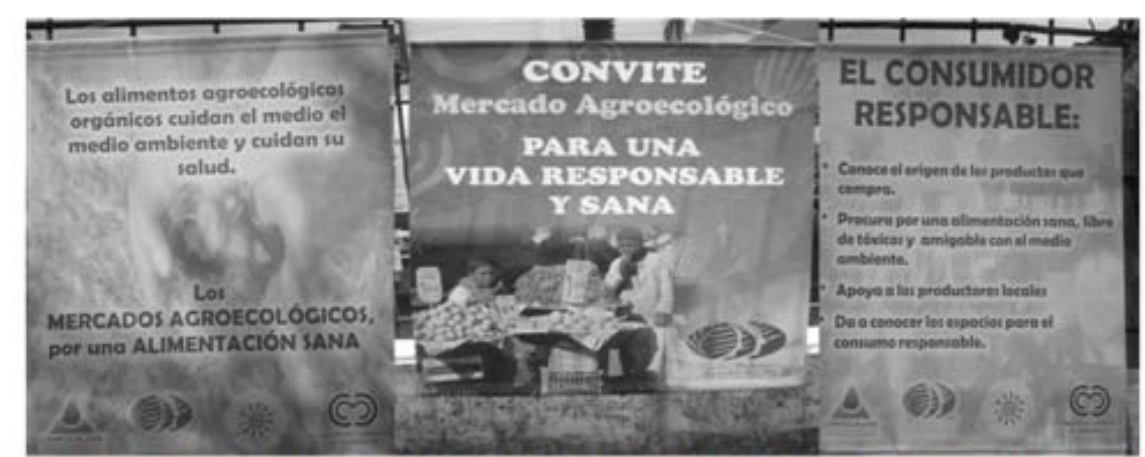

Figura 2. Pendones alusivos al Mercado Agroecológico asumidos como material de análisis.

Una vez transcritas las entrevistas, repasado el diario de campo y compilado el material visual y escrito, se siguieron los siguientes pasos para el análisis de la información: a) lectura repetitiva de los textos, b) búsqueda de temas emergentes, c) construcción de categorías relacionales, d) disgregación de información atendiendo a su pertinencia de acuerdo con las distintas categorías construidas, y e) análisis e interpretación de resultados.

Finalmente, fundamentado en la premisa de que las prácticas de consumo constituyen un acto comunicativo (¿De qué habla el consumo agroecológico en términos del consumidor y su relación con los otros, consigo mismo y el medio social en que se desenvuelve cotidianamente?), la presentación de resultados se organizó en función de cuatro categorías relacionales surgidas del análisis de contenido. Estas son: a) consumo agroecológico y responsabilidad socioambiental, b) consumo agroecológico y práctica ciudadana, c) consumo agroecológico y 
diferenciación identitaria, d) consumo agroecológico y afectividad, y e) consumo agroecológico y evocación sensorial, guía que orientará el siguiente apartado.

\section{RESULTADOS Y DISCUSIÓN}

Sin desconocer que las múltiples narrativas empleadas para justificar la opción por alimentos agroecológicos no son exclusivas de uno u otro tipo de consumidor, sino que, en la práctica, todas o algunas podrían ser conjuntamente empleadas por un mismo individuo o grupo, en aras de una descripción más didáctica, a continuación se abordan separadamente, usando como guía las categorías construidas.

\section{Consumo agroecológico y responsabilidad socioambiental}

En lo referente a las narrativas que sustentan la opción por alimentos ecológicamente producidos, entre la mayoría de los entrevistados prima la siguiente relación: el asocio entre sus prácticas de consumo y la idea de responsabilidad socioambiental. Una forma de justificar para sí mismo la elección de dichos alimentos es la búsqueda de alternativas a la producción agroindustrial. En términos generales, optar por una "alimentación natural" u "orgánica", nociones comúnmente manifiestas por los entrevistados, surge como respuesta primaria a los temores y ansiedades generadas en torno a los procesos productivos cimentados en el uso de agroquímicos (principalmente insumos de síntesis empleados en la fertilización y control fitosanitario de cultivos), asunto reflejado en el siguiente testimonio típico:

Hoy uno no sabe qué es lo que se come. Aunque uno no pueda verlo, en un tomate hay residuos de agroquímicos, igual en una lechuga $o$ en cualquier otra hortaliza. ¿Cuántas enfermedades no serán producidas por eso sin que uno no lo sepa? Por eso lo mejor es comer productos limpios, no contaminados, productos mucho más naturales [...] (Laura Carmona, consumidora ocasional)

Al interior del grupo estudiado, la antítesis general observada corresponde a natural/agroecológico en contraste con artificial/agroquímico, antinomia que orienta la opción por un tipo de alimentos producidos a partir de 
procesos "más limpios", con su consecuente menor impacto ambiental. Analíticamente, tal resultado permite dilucidar la oposición simbólica entre pureza e impureza (Douglas, 1998), donde lo impuro corresponde a aquellas prácticas productivas asumidas como contaminantes y degradantes de la salud humana y los recursos naturales, en contraste con aquellas vistas como "más amables con el medio ambiente". Para estos consumidores, "evitar contribuir al deterioro del planeta", mediante la opción consciente por alimentos agroecológicos, constituiría una acción crítica y restauradora del equilibrio ambiental hoy puesto en riesgo. Se trata de una opción fundamentada en el rechazo a lo impuro, esta vez específicamente concretada a partir de la adquisición e incorporación a la dieta de "alimentos sanos", es decir, poseedores de atributos positivamente valorados como ser producidos a pequeña escala (en lo posible por comunidades campesinas), no presentar trazas de agrotóxicos, ser fertilizados con biocompuestos, entre otros:

Cuando yo compro y como un alimento limpio, siento que estoy un poco más en paz con el planeta. No es sólo cuestión de beneficiarme yo, sino de cuidar el planeta, comprándole a quienes también lo cuidan, a quienes producen sin químicos ni ningún otro veneno contaminador [...] (Antonio Reinosa Henao, consumidor ocasional)

En este caso, "poner un granito de arena para la conservación del medio ambiente" representa una forma, bien sea explícita o implícita, de manifestar en el quehacer cotidiano responsabilidad socioambiental. A partir de acciones y elecciones individuales, los consumidores se asumen, hasta cierto punto, corresponsables del proceso de conservación/degradación ambiental, siendo la opción por consumo ecológico una respuesta a ello. En este contexto, el hecho de asistir al Mercado Agroecológico, sobre todo cuando se hace como consumidor constante, aparece como una forma de materializar el deseo individual de contribuir a la construcción de modelos agroproductivos distintos a los dominantes, tal vez confiando en que la suma de estos actos aislados/personales contribuyan a la resolución de un asunto de carácter público: la degradación medioambiental.

En última instancia, se trata de prácticas individuales conscientes, norteadas por valores de orden ambientalista, que dan cuenta de la intención de avanzar en dirección de una utópica sociedad sustentable, donde el consumo agroecológico constituye, entre otras, una estrategia 
política para ello, asunto revelado por el estudio y presentado a continuación.

\section{Consumo agroecológico y práctica ciudadana}

Para la mayoría de integrantes del grupo estudiado, la opción por el consumo de productos agroecológicos, además de constituir un acto individual de responsabilidad ambiental, representa una forma de ejercicio ciudadano, en este caso entendido como una actividad capaz de transformar, de forma pausada y escalonada, estructuras políticas y sociales dominantes que producen exclusión, desigualdad e inequidad. Testimonios recurrentes, como el enseguida expuesto, sustentan esta aseveración:
Las políticas de la revolución verde, que están relacionadas con la misma explotación del petróleo y con otros problemas mundiales de carácter ambiental, desequilibran nuestro ambiente, por lo que no se deben de apoyar su producción, optando por consumir productos no contaminantes, es decir, productos más amables con el medio ambiente. Esta idea es la que yo comparto con las otras personas que vienen a acá [al merado]. Así sea en pequeño, aquí se está haciendo algo para cambiar el modelo [...] (Claudia Escobar Zuluaga, consumidora permanente)

En este marco, más allá de un espacio de mero intercambio comercial, analíticamente, el Mercado Agroecológico puede ser significado como aquel espacio físico, político y moral de encuentro que motiva y posibilita la convivencia y la interacción social entre actores individuales comocolectivos, entre consumidores y productores agroecológicos, unidos en el intento de desarrollar dinámicas socioeconómicas alternativas centradas en principios de solidaridad, justicia y corresponsabilidad socioambiental. En términos concretos, interpretativamente, este espacio es asumible como un medio afín al interés de transformar la lógica capitalista de mercado, constituyendo un escenario de intercambio más equitativo e incluyente, manifiesto en la intención de circunscribir y resarcir a aquellos actores históricamente en desventaja: los productores campesinos y familiares, generalmente vulnerados al interior de las cadenas productivas y de formación de precios, para el caso estudiado. 
Aunque, debido a las dimensiones del Mercado Agroecológico, lo que se pueda hacer en términos reales aún es poco, tal como hoy puede ser observado, este espacio constituye, sobre todo, un punto o nodo de educación ciudadana:

Yo entré aquí un día por pura curiosidad. Pero luego vi que la cosa aquí es distinta. Uno puede hablar con quienes venden, que son los mismos que producen, muchos de ellos campesinos. Aquí uno puede aprender de ellos, de cómo ellos hacen las cosas. Yo no sabía mucho sobre la producción ecológica, sobre la agricultura limpia, sobre la variedad de semillas que existen y que hoy se están perdiendo, por ejemplo. Fue aquí donde aprendí estas cosas, valiosas para aprender y compartir (Robinson González Marín)

Iniciativas de este tipo procuran ilustrar a otros consumidores acerca de las prácticas injustas de comercio presentes a lo largo de las cadenas de mercado; los efectos de los alimentos producidos convencionalmente sobre la salud humana y ambiental; las consecuencias sociales y ambientales del monocultivo como las de producción agrícola industrial; asimismo del valor de los alimentos agroecológicamente producidos, la biodiversidad y la conservación de los recursos. En lo cotidiano, circulando por los distintos puestos del mercado, conversando aquí y allá con consumidores y productores, es posible participar de pláticas sobre alimentación saludable, medio ambiente, crítica a los modelos de producción "tipo revolución verde", entre otros temas próximos a la propuesta agroecológica.

Sustentado en lo anterior podría asumirse que espacios como el descrito, es decir, en los que consumidores y productores comparten entre ellos y con otros parte de aquellas narrativas que soportan su opción agroecológica, constituyen escenarios de formación y educación ciudadana en torno a la cuestión socioambiental. Se trata de espacios en que se hace ver al otro que acciones tomadas en el ámbito privado (en este caso el acto cotidiano de decidir qué comprar, dónde y a quién) repercuten sobre la esfera pública, bien sea para transformar el orden social predominante o para mantenerlo y reforzarlo.

Conductas como las señaladas corresponderían a acciones pro-consumo responsable, orientadas a construir una nueva cultura de acción política a partir de las prácticas de 
consumo. En este sentido, acorde con la propuesta de transformación social defendida, el perfil de los "consumidores agroecológicos" correspondería al de actores politizados alrededor de la causa conservacionista y ambiental. Adoptando la caracterización propuesta por Portilho (2009), se trataría de actores con gran envolvimiento con cuestiones socio-ambientales, que se autoatribuyen responsabilidades y deberes en relación con el medio ambiente y la transformación social, que prefieren actuar en la espera del consumo, más que en acciones políticas convencionales.

En términos generales, actuar en consecuencia con esta ideología de consumo constituye, para quienes lo hacen, un referente identitario que da cuenta de una forma de ser y actuar particular, impronta usada por algunos, como se expondrá a continuación, como elemento de distinción o diferenciación social en un contexto que permite, vía contraste, reconocer la diferencia.

\section{Consumo agroecológico y diferenciación identitaria}

Para quienes optan por el consumo de alimentos agroecológicos, el consumo de este tipo de productos, en términos generales, constituye una forma de expresar quién se es. En tal sentido, podría plantearse que su opción de consumo constituye en sí una intención comunicativa: demostrar lo que se es frente a lo que no se es, creando una definición propia basada en la diferencia. Siguiendo esa línea, para la mayor parte de los entrevistados, ser "consumidor agroecológico" constituye un referente de identidad:

Cuando uno viene aquí al mercado, uno se siente en familia. Aquí no sólo somos vendedores y compradores, sino también amigos. Uno aquí se encuentra con gente que conoce, que es más o menos como uno, o sea, que está preocupado por el cuidado del ambiente, por el desarrollo sostenible. Son principios que casi todos compartimos, es como si todos tuviéramos puesto, algo así como la misma camiseta [...] (Daniel Salazar Ríos, consumidor permanente)

Como fue constatado en campo, la opción, la preferencia y/o la afinidad por el consumo de alimentos agroecológicos sirve para saber quién es compatible con quien y hasta dónde se puede establecer una relación con el otro, basada en la tenencia compartida de ciertas cualidades, actitudes e ideas: la preocupación ambiental, la valoración del trabajo 
campesino, el rechazo a ciertas tecnologías consideradas riesgosas (las semillas y los cultivos transgénicos, como preocupación contemporánea), la censura a la dominancia del modelo capitalista de mercado, el respeto a los conocimientos ancestrales, el uso de tecnologías "limpias" y el "reaprovechamiento de recursos", entre los temas destacados entre los entrevistados.

En este contexto, saber con quiénes se es o no compatible da pie al establecimiento de relaciones entre actores, bien sea de carácter solidario o conflictivo, que refuerzan dicha identidad. Un caso que destaca en el entorno de estudio es la presencia del Grupo de Estudios en Agriculturas Sustentables (GEAS), grupo adscrito al Jardín Botánico de la Universidad de Caldas, que congrega y crea hermandad entre estudiantes de distintas disciplinas en torno a la propuesta agroecológica, y que, desde su concepción tecnológica, científica e ideológica, soporta logística y conceptualmente el Mercado Agroecológico. Hacer parte de este grupo constituye en sí una forma de expresar desacuerdo y distanciamiento con relación a la producción agrícola "convencional", es decir, la basada en el uso de insumos agroquímicos, el monocultivo y esquemas administrativos de orden empresarial/agroindustrial, opción que los distingue de otros actores acríticos, afines o alineados frente a estas formas de producción:

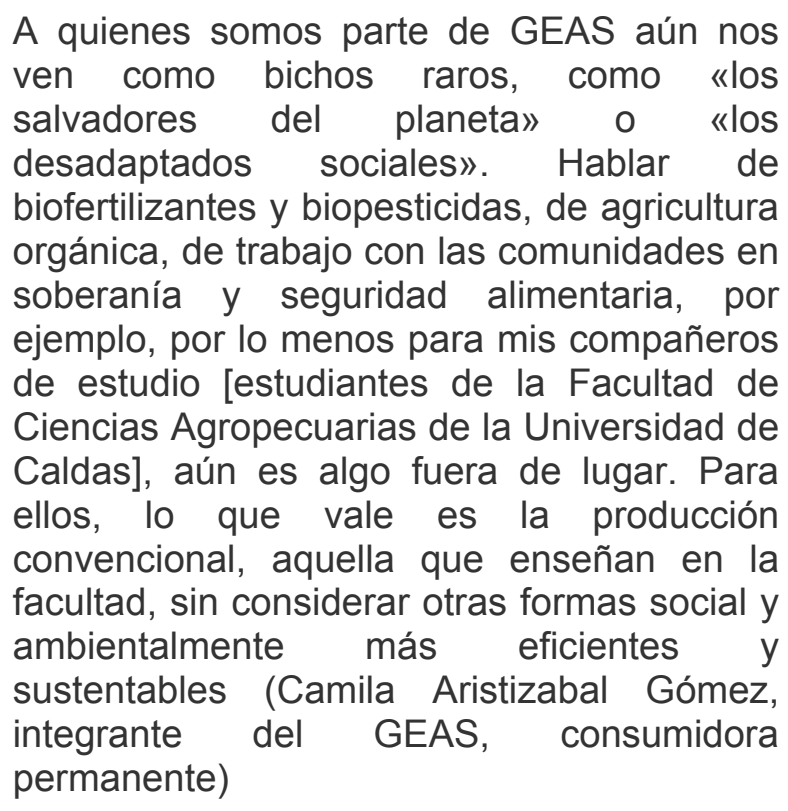

Tal como se ilustra en el anterior testimonio, asumir una determinada identidad exige aceptar la aprobación o la desaprobación de los otros, en medio de un juego de relaciones de poder. En este caso, el establecimiento de relaciones conflictivas, esta vez manifiestas a partir de la desautorización del otro, recrea y refuerza las identidades 
en disputa. El reconocimiento de la existencia de unos "otros" alternativos (de "los agroecológicos", específicamente) por parte de quienes los conciben diferentes, sustenta la distinción en doble vía. En este sentido, el acto de producir y consumir alimentos agroecológicos, en un contexto en que la producción basada en el uso de insumos de síntesis, comunica a los otros actores elementos y criterios de distinción social, valiéndose de códigos reconocidos y entendidos por quienes los emiten y reciben. Se trata de códigos que, a partir de narrativas y pautas de comportamiento, expresan lo que se es, lo que no se es y cómo serlo.

En síntesis, el consumo agroecológico (como cualquier otra condición de consumo) puede ser analizado como una forma de lenguaje (Douglas, 1998) usada para marcar diferencias y reafirmar identidades, esta vez desarrolladas en torno a la responsabilidad socioambiental y a la vivencia de valores de justicia y solidaridad social, convicciones manifiestas en el escenario público, que se cruzan con las exteriorizadas en un ámbito más privado o personal, asunto a tratarse a continuación. Separándonos un poco del escenario colectivo ¿Qué expresa el lenguaje del consumo agroecológico en un espacio más doméstico, interpersonal y familiar?

\section{Consumo agroecológico, afectividad y cuidado}

Entre las principales narrativas usadas por los consumidores para justificar su opción por los alimentos agroecológicos aparecen, recurrentemente, las asociadas al autocuidado y el cuidado de otros. Lo hallado en campo revela padres preocupados con los riesgos inherentes al uso de pesticidas, hormonas, colorantes y conservantes, entre otros insumos empleados en la producción de alimentos, tal como se refleja en el siguiente testimonio:

Yo comencé a consumir productos agroecológicos debido a toda la información que conozco sobre los daños que causan a la salud los alimentos que contienen residuos de pesticidas y sustancias conservantes. Cada vez que vengo acá, compro alimentos y los consumo en casa, me siento más sana y vigorosa. También siento una alegría inmensa porque sé que estoy cuidando a mi familia. Siempre trato de darles lo mejor, pues siempre quiero lo mejor para ellos, cuidarlos y consentirlos. Para mí, saber que los niños están comiendo sano, creciendo y desarrollándose bien, evitando enfermedades, es una tranquilidad inmensa 


\section{[...] (Rosalba Dávila López, consumidor permanente)}

En casos típicos como el anterior, la preocupación por el cuidado propio y el de otras personas a cargo puede interpretarse como un acto de afecto. Hoy día, lo que en algún momento podría haberse visto como ventajas de la industrialización (facilidad de preparación de los alimentos, posibilidad de vencer la estacionalidad mediante el uso de conservantes, alargamiento de la vida útil de frutas y verduras), cobra un nuevo sentido ligado al riesgo y la incertidumbre ¿Cómo saber cuál será el efecto a largo plazo del consumo de alimentos con residuos de pesticidas, colorantes artificiales y un sinnúmero de aditamentos químicos?, se cuestiona a varios de los entrevistados.

Tal como se corroboró en campo, los riesgos asociados al tipo de alimentos incorporados a la dieta son hoy más que nunca causantes de ansiedad entre los actores que hicieron parte del estudio. La presencia de trazas de agroquímicos, el uso excesivo de aditivos conservantes, el alto contenido de grasas, sal y azúcar, entre los principales elementos señalados, justifican la opción por alimentos agroecológicos, en donde elegir lo mejor para los hijos, familiares y allegados más próximos constituye un acto afectivo manifiesto en la provisión de bienestar, y protección.

En este contexto, la investigación muestra que brindar cuidado de esta forma implica casi siempre recurrir a los principios de precaución y prevención:

Uno no puede ser tan confiado, uno no sabe los efectos de los alimentos que come. Ahora vemos que la mayoría de productos son dañinos, como es el caso de los transgénicos o el de los pollos levantados usando todo tipo de hormonas. Uno no tiene certeza de esos daños, pues unos dicen que sí y otros que no son dañinos, pero lo mejor es prevenir, optar por lo natural, lo que no afecta a las personas y al ambiente. Lo que yo más deseo es sentirme tranquila y en paz, por eso elegí los alimentos orgánicos [...] (Jenny Joanna Penagos, consumidora ocasional)

En el escenario descrito, el consumo de alimentos ecológicamente producidos representa una estrategia para disminuir los efectos de ingerir productos valorados como 
contaminadores o "impuros". La precaución frente a los riesgos potenciales y la defensa ante aquellos que han sido comprobados justificaría la opción por lo agroecológico, en términos del cuidado propio y el de los otros. Podría plantearse que, por lo menos para estos actores, la toma de conciencia acerca de los daños producidos por algunas técnicas y tecnologías agropecuarias sobre el entorno natural y, en consecuencia, sobre la salud humana, animal y vegetal, conduciría a tomar una postura reflexiva moral frente a ello. Dicha lectura moral del problema llevaría a los consumidores a evitar vivir con éste como si nada estuviera pasando, situación que igualmente justifica su elección agroecológica preferencial

En un escenario de escases, contar con quienes producen y directamente ofertan este tipo de alimentos constituye un avance en pro de dar respuesta a las intenciones y preocupaciones declaradas, hecho que sustenta la gratitud y el aprecio de los consumidores hacia aquellos productores que se esfuerzan por producir de manera agroecológica, poniendo en manifiesto otra relación que articula afectividad y consumo agroecológico. Empíricamente, en escenarios como el Mercado Agroecológico es posible constatar y percibir encuentros cargados de sensibilidad entre las partes; trato amable y familiar entre quienes venden y adquieren los productos, alegría por el encuentro con amigos y conocidos, interés en los expuesto por quienes dan explicaciones sobre lo que es un producto agroecológico, entre otras situaciones. Dichas observaciones permiten argumentar que, los alimentos agroecológicos, además de ser vistos por los consumidores como bienes ambiental y políticamente producidos, son vislumbrados como unidos a personas concretas, sujetos con quienes es posible interactuar y establecer lazos de afectividad, solidaridad y confianza; relaciones que van mucho más allá de simples transacciones comerciales.

Dando un salto con relación a lo recorrido hasta ahora, la investigación también permitió establecer que no todos los consumidores de alimentos agroecológicos sustentan su opción por este tipo de productos en criterios cuyos pilares a grandes rasgos son la sustentabilidad ecológica, económica y social. Como se analizará a continuación, otros motivos de orden más insustancial (en términos de una propuesta de transformación socioeconómica) informan y orientas esa misma elección.

\section{Consumo agroecológico y expectativas alimenticias}

Para parte de los consumidores indagados, la opción por alimentos agroecológicos se sustenta en la oportunidad de descubrir nuevos productos que respondan a sus 
expectativas alimenticias: alimentos que previenen enfermedades, mejoran el funcionamiento corporal y "evitan el envejecimiento", por ejemplo, perspectiva descrita en el siguiente testimonio típico:

Para mí consumir alimentos orgánicos hace parte de seguir un estilo de vida saludable. Lo que encuentro en éstos es mejores contenidos nutricionales, mayor calidad debido al no uso de agroquímicos. Vengo al mercado [agroecológico] porque aquí en Manizales es uno de los pocos lugares que pueden conseguirse. De vez en cuando consigo algunas hortalizas en [el supermercado] Carrulla, aunque sé que un sábado al mes puedo conseguirlos aquí [...] (Antonio Giraldo Ortiz, consumidor permanente)

En estos casos, al momento de elegir qué tipo de alimento comprar, valores y propósitos como la responsabilidad ambiental, la solidaridad y el compromiso ético con los productores, la apuesta por un comercio más justo y equitativo, la intención de avanzar hacia un nuevo modelo socioeconómico sustentable, el ánimo de apoyar los movimientos y organizaciones agroecológicos, entre otros posibles criterios, no juegan un papel destacado, incluso llegando a no tener cabida. Se trata de un interés individual por la "alimentación sana", argumento totalmente válido como narrativa de consumo, que, a diferencia de los anteriormente analizados, no le apuesta a una transformación social más colectiva y envolvente.

Para estos consumidores, la calidad organoléptica y el valor nutricional representan dos de las principales variables orientadoras de su opción por este tipo de productos. Reconocer nuevos sabores, colores y texturas; propiedades medicinales y nutricionales por ellos antes desconocidas, así como condiciones higiénicas y de inocuidad atribuibles a su estado natural, entre otras características, respalda su decisión de preferirlos sobre otros alimentos disponibles. Dicha opción tendría sustento en un salto conceptual que asocia "natural" con "saludable", por un lado, y "agroecológico" con "más nutritivo y especial", por otro. Se trata de asociaciones más o menos comunes, construidas sobre todo a partir de las informaciones circulantes por distintos medios de comunicación masiva, usualmente aprendidas por los consumidores como algo dado por sentado o cierto:

Quise experimentar con los productos agroecológicos buscando mantener un buen 


\begin{abstract}
estado de salud. Primero me interesé por varios programas que vi en televisión sobre la importancia de los alimentos: <somos lo que comemos>, en los que recomendaban consumir productos orgánicos y agroecológicos. Luego me fui interesando más, buscando información en internet, averiguando dónde podía conseguirlos en la ciudad [Manizales], hasta que di con el de la Universidad de Caldas [...] (Luisa Ortiz, consumidor permanente)
\end{abstract}

En este caso, la información brindada por medios considerados fiables orienta la opción por este tipo de alimentos. La proliferación de programas radiales y televisivos dedicados a temas de salud, que además cuentan con un público cautivo, coadyuvan a crear inquietud entre los consumidores acerca de la existencia de productos alternativos, entre los que figuran los alimentos agroecológicos. Como fue corroborado en campo, para varios de los entrevistados, éstas fueron las fuentes iniciales de información, posteriormente complementadas con el descubrimiento de la existencia de un Mercado Agroecológico local, que además cuenta con el apoyo institucional de una universidad de amplio reconocimiento social.

En este caso, la fiabilidad de la condición agroecológica de los alimentos adquiridos, más que de la confianza resultante de la relación próxima y el conocimiento mutuo entre consumidores y productores, deriva del respaldo brindado por el Jardín Botánico de la Universidad de Caldas, en esta ocasión prácticamente asumido como marca indicadora de confianza y garantía de autenticidad. En dicho marco, al optar por este tipo de alimentos, los consumidores buscarían cubrir necesidades principalmente funcionales, donde el valor subjetivo otorgado por el comprador estaría determinado tanto por la utilidad del producto (brindar salud y bienestar corporal, en este caso) como por la certeza de estar adquiriendo un producto auténtico (auténticamente agroecológico e informalmente certificado por la Universidad de Caldas) que cumple con sus expectativas de consumo.

En términos generales, lo anterior permite argumentar que si bien hay siempre un imaginario asociado a la adquisición de un producto, éste no siempre es el mismo para todos, hecho que lleva a prestar mucha atención a la heterogeneidad aportada por cada situación particular. En este marco, lo que viene a continuación es una muestra más de la premisa señalada, donde más allá del consumo funcional y de las ideologías alimentarias de índole político 
y socioambiental, la experiencia sensorial también ocupa un lugar privilegiado para algunos.

\section{Consumo agroecológico y evocación sensorial}

Atendiendo a la premisa anterior, la investigación permitió señalar que, si el interés consiste en comprender las narrativas con la que los actores sociales justifican sus opciones de consumo, adentrarse en escenarios más íntimos y subjetivos, explorando todo aquello que desata "llevar un alimento a la boca", constituye también un camino a seguir:

Si uno manda comprar un huevo a la tienda - lo compra en el supermercado, es un huevo blanco, sin ningún sabor, producido en galpones donde hay miles de gallinas, alimentadas a base de purina y todos esos productos procesados. En cambio, cuando uno viene aquí al Mercado, hasta encuentra huevos de colores, huevos criollos que saben muy bueno, de gallinas bien alimentadas con maíz, plátano y hierbas. Esos huevos los disfruto mucho, pues son como los que mi mamá y mi abuela cogían y nos daban en la finca, como los que comíamos cuando niños, huevos con el sabor del campo, como los que uno se comía en esos buenos tiempos [...] (Rosalba Dávila López, consumidora ocasional)

Como ilustra el anterior testimonio, para algunos consumidores, percibir un sabor distinto o diferenciable, esta vez asociado al de los productos agroecológicos, puede inducir a un estado afectivo y emocional, asumible, como un valor hedónico positivo placentero o positivo. Recuperar sensaciones del pasado, "el mismo sabor de las frutas que me daban mis tías cuando íbamos de visita a la finca cuando niños", por ejemplo, impresiones que, objetiva o subjetivamente, asocian este tipo de alimentos a hechos que evoquen recuerdos agradables, justifica su predilección.

Lo anterior permite constatar que los alimentos que se comen tienes historias asociadas con el pasado de quienes los ingieren (Mintz, 2003). En el caso de estudio, evocar un pasado rural, cuando hoy se vive en un entorno citadino; recordar lo producido en la finca donde se nació y de la que luego se salió, en la que se producía sin aditamentos químicos y se mantenía una huerta familiar para el autoconsumo, por ejemplo, darían cuenta de esa memoria 
sensorial que asocia pasado, gusto y sensaciones memorables. Analíticamente, consumir este tipo de alimentos constituiría una forma de anclaje que satisface cierto deseo de arraigo y regreso al "terruño", a partir de un imaginario ligado al mundo rural recordado y tal vez idealizado. En este contexto, la disponibilidad y el consumo de alimentos agroecológicos brinda una oportunidad, no desaprovechada, de revivir buenos momentos mantenidos en la memoria y el imaginario de ciertos consumidores, remembranzas que en este caso reaparecerán bajo el estímulo gustativo.

En síntesis, podría decirse que, en todas las narrativas encontradas para justificar el consumo de alimentos agroecológicos, por encima del valor nutricional de uso, más que de un alimento en sí, de lo que los consumidores se apropian es de lo que éste evoca, presupone y simboliza, tal como fue expuesto a lo largo del artículo.

\section{CONCLUSIONES Y CONSIDERACIONES FINALES}

- El estudio permite concluir que, por lo menos para casos afines al contexto en que se enmarca el mismo, más que como un simple acto de compra y uso de bienes, el consumo de alimentos agroecológicos debe ser entendido como un proceso sociocultural de apropiación de significados y valores contenidos en o atribuidos a cada producto adquirido. En esta vía, como fue enfatizado a lo largo del artículo, la ingesta de este tipo de alimentos no satisface apenas necesidades fisiológicas, sino también de índole comunicativa, simbólica e indentitaria.

- Aunque el imaginario dominante hoy puede girar alrededor de la responsabilidad social, la protección ambiental y la salud, es preciso reconocer que éste no es estático e inmutable. Como se pretendió demostrar en torno a su consumo, diferentes lógicas, justificativas y racionalidades pueden coexistir, incluso en el pensamiento de un mismo sujeto, siendo esta una consecuencia de la heterogeneidad social prevaleciente: distintos actores significando un aparente mismo bien desde distintas perspectivas.

- En términos generales podría plantearse que cuestiones motivadoras del consumo agroecológico, tales como, solidaridad con los pequeños productores, evocación de recuerdos agradables, cuidado y auto-cuidado, crítica a la producción agroindustrial, ansias de autoafirmación y pertenencia a un grupo, entre otras narrativas repasadas, derivarían de cambios de índole global operados en el clima social contemporáneo. Asuntos como la degradación de los recursos naturales, la dominancia de sistemas productivos que privilegian lo económico sobre lo socioambiental, el 
abuso de agroquímicos en la producción de alimentos, la pérdida de sentido del bienestar colectivo (ante la dominancia del individualismo), entre otras problemáticas actuales, podrían estar informando y orientando las opciones agroecológicas de consumo.

- Finalmente, apoyado en lo expuesto por Canclini (1995), vale decir que, en términos generales, trabajos como el presente son una oportunidad para ver el acto de consumir como algo que supera la mera respuesta a gustos, antojos y compras hechas porque sí, premisa que invita a asumir el consumo como un proceso sociocultural en la que los actores sociales, a partir de sus imaginarios $y$ representaciones, se apropian de lo que compran y consumen otorgándoles significados y usos particulares.

\section{BIBLIOGRAFÍA}

- Barbosa, L. \& Campbell, C. (2006). O estudo do consumo nas ciências contemporâneas. Em: Cultura, consumo e identidade. Lívia Barbosa y Colin Campbell (org.). FGV Editora. Rio de Janeiro.

- Blee, K. (1985). Family patterns and politicization of consumption. Sociological Spectrum. 5 (4): 295-316.

- Castañeda, M. (2010). Ambientalização e politização do consumo e da vida cotidiana: uma etnografia das práticas de compra de alimentos orgânicos em Nova Friburgo/RJ. Tesis de Maestría. Río de Janeiro, Universidad Federal de Río de Janeiro-Instituto de Ciencias Humanas y Sociales.

- Campbell, C. (2006). Eu compro, logo existo: as bases metafísicas do consumo moderno. Em: Cultura, consumo e identidade. Lívia Barbosa y Colin Campbell (org.) FGV Editora. Rio de Janeiro.

- Cohen, M. (2001). The emerging international policy discourse on sustainable consumption. En: Cohen, M. \& Murphy, J. (Eds.). Exploring sustainable consumption: environmental policy and social science. Oxford: Elsevier Science. pp. 21-38.

- Douglas, M. (s.f.). Pureza e perigo. Editora Perspectiva, São Paulo.

- Douglas, M. \& Ischerwood, B. (2006). O mundo dos bens: para uma antropologia do consumo. Rio de Janeiro: Ed. UFRJ.

- García-Canclini, N. (1999). Consumidores e cidadãos. Editora UFRJ. Rio de Janeiro.

- Guhl, Andrés. (2009). Café, bosques y certificación agrícola en Aratoca, Santander. Revista de Ciencias Sociales. No 32., pp. 114-125.

- Jodelet, D. (2006). Place de l'expériencie vécue dans les processus de formation des représentations sociales. En: Haas, V. (comp.). Les savoirs du quotidien. PUR, París. 
- Liden, M.. (1994). Working class consumer power. International Labor and Working-class History. No 46., pp. 109-121.

- Méndez, C. (2007). Comunicación e identidad: una introducción al estudio del consumo. Universitas Humanística. No 64., pp. 291-305.

- Miller, D.. (2002). Teorias das compras. O que orienta as escolhas dos consumidores. Editora Nobel. São Paulo.

- Mintz, S.. (2003). Sabor a comida, sabor a libertad. Incursiones en la comida, la cultura y el pasado. CIESASCONACULTA. México.

- Parrado, Á.; Montoya, I. (2007). Características del consumo de vegetales en los estratos socioeconómicos medio y alto de Bogotá. Agronomia Colombiana. No 2., Vol. 25, pp. 377382.

- Portilho, F. (2005). Sustentabilidade ambiental, consumo e cidadania. Editorial Cortez. São Paulo.

- . (2009). Sociabilidade, confiança e consumo na feira de produtos orgânicos. Em: Barbosa, L.; Portilho, F.; Veloso, L. (orgs). Consumo: cosmologias e sociabilidades. Mauad, Rio de Janeiro. pp. 61-86.

- Stolle, D.; Hooghe, M.; Micheletti, M. (2005). Politics in the supermarket: political consumerism as a form political participation. International Political Review 26(3): 245-269.

- Warde, A. (2005). Consumption and theories of practice. Journal of Consumer Culture. No 2., Vol. 5., pp. 131-153.

1. Ingeniero Agrónomo. Magister en Desarrollo y Sociología Rural. Doctor en Desarrollo, Agricultura y Sociedad. Docente adscrito al Departamento de Desarrollo Rural y Recursos Naturales de la Universidad de Caldas. Miembro del Grupo CERES (Centro de Estudios Rurales) y del Instituto de Ciencias Sociales y Humanas (ICSH) de la misma institución. e-mail: marlon.mendez@ucaldas.edu.co

2. Ingeniero Agrónomo. Miembro del semillero de investigación en Ruralidades Emergentes, Departamento de Desarrollo Rural y Recursos Naturales. Facultad de Ciencias Agropecuarias, Universidad de Caldas. e-mail: fausto.bonilla@hotmail.com 